\title{
A cross-sectional study of hypertensive outpatients to determine the necessity of asking about erectile dysfunction symptoms.
}

\begin{abstract}
Background: Erectile dysfunction (ED) is common amongst hypertensive men. Hypertensive patients often attribute it to antihypertensive drugs, although conflicting evidence linking ED with antihypertensive medication exists. The objectives were to determine the prevalence and severity of ED, the type of treatment sought, and the risk factors for ED among hypertensive men. Method: A cross-sectional survey conducted over six months from June to November 2008 at University Kebangsaan, Malaysia Medical Centre, Kuala Lumpur. Inclusion criteria included hypertensive men above 30 years old, with essential hypertension for at least three months. We excluded diabetics, a history of pelvic surgery and known psychiatric illnesses. The International Index of Erectile Function-5 (IIEF-5) assessment was used with a standardised checklist. We analysed data using SPSS, to assess the prevalence and association of ED with selected variables. Results: Of the 200 participants screened, 35.5\% perceived that they had ED. However, prevalence increased to $69 \%$ after screening using an IIEF-5 questionnaire. Forty-eight per cent were reported to have moderate-tosevere ED. ED was significantly associated with age $(p$-value $=0.0001)$. No significant associations were found between ED and the duration of the hypertension ( $\mathrm{p}$-value $=0.505$ ), hypertension control ( $p$-value $>0.05$ ), smoking status ( $p$-value $=0.858$ ) or number of antihypertensive medication taken ( $\mathrm{p}$-value $>0.05$ ). Among perceived and proven ED patients, traditional medicines were mainly used for treatment (18.3\% and $17.2 \%$ respectively). Conclusion: ED is a problem among hypertensive patients. It was associated with age but not with hypertension duration, control, number of antihypertensive drugs or smoking. Physicians should enquire about ED symptoms in hypertensive patients, as most of them resorted to selftreatment with traditional medicines.
\end{abstract}

Keyword: Erectile dysfunction; Follow-up; Hypertension. 